\title{
Líneas de investigación aplicables al Programa de Comercio Internacional de la Universidad Francisco de Paula Santander en la ciudad de Cúcuta ${ }^{1}$
}

\author{
Research lines applicable to the International Trade Program \\ from Francisco de Paula Santander University Cucuta city
}

Liliana Marcela Bastos Osorio ${ }^{2}$

\begin{abstract}
Resumen
Este artículo presenta los resultados de una investigación cuya finalidad es construir líneas de investigación aplicables al Programa de Comercio Internacional de la Universidad Francisco de Paula Santander UFPS. Esta investigación fue de carácter descriptivo y de campo. Descriptivo porque se hizo la revisión de los criterios de investigación del Proyecto Educativo del Programa PEP del Programa de Comercio Internacional y se hizo una observación a las diferentes líneas de investigación de los programas afines a Comercio Internacional. De campo porque se aplicó una encuesta a una muestra del sector externo empresarial de la ciudad de Cúcuta, y a los docentes del Programa de Comercio Internacional de la UFPS. El resultado fue la consolidación de diez líneas de investigación para el Programa de Comercio Internacional.
\end{abstract}

Palabras clave: Línea de investigación; Comercio internacional; Universidad Francisco de Paula Santander.
This article presents the results of an investigation addressed to build lines of research applicable to the program of International Trade from Universidad Francisco de Paula Santander University UFPS. The nature of this study was descriptive and field research. Descriptive, since the research criteria of the Program Educational Project (PEP) of the International Trade Program was revised as well as an observation to the different $\mathrm{l}$ ines o $\mathrm{fr}$ esearch $\mathrm{r}$ elated $\mathrm{t}$ o I nternational Trade programs was made. Field research, since a survey was administered to a sample of corporate external sector of Cucuta city as well as to professor of the UFPS International Trade program. The result was the consolidation of ten lines of research for the International trade program.

Keywords: Line of research; International trade; Universidad Francisco de Paula Santander.

\footnotetext{
${ }^{1}$ Este artículo es resultado de un proyecto de investigación con aval institucional y financiación de la Universidad Francisco de Paula Santander UFPS, Cúcuta.

2 Magister en Gerencia de Empresas - Universidad Nacional Experimental del Táchira (San Cristóbal, Venezuela), Profesor Tiempo Completo Departamento de Estudios Internacionales y de Fronteras, Universidad Francisco de Paula Santander, Cúcuta. Investigador del Grupo de Investigación para el Desarrollo Socioeconómico - GIDSE, categorizado por Colciencias en A (2015). E-mail: lilianamarcelabo@ufps.edu.co
} 


\section{Introducción}

El espacio de las líneas de investigación responde al espíritu de formación de los estudiantes que presentan una mente abierta a los cambios sociales, económicos, y a la necesaria vinculación que tiene el programa de Comercio Internacional UFPS en la aproximación a dichas evoluciones a la luz de las reflexiones que desde esta se gestan, lo que implica un necesario encuentro desde lo interdisciplinar.

Teniendo en cuenta que la investigación en líneas como un sistema, constituye instancias de construcción de conocimiento y ello a la vez permite retroalimentar el currículo, las reflexiones que se suscitan desde las líneas de investigación como núcleos problémicos son las que abren paso a la circulación permanente de los saberes dados en los diferentes bloques programáticos de los ciclos de los programas educativos (García y Núñez, 2006).

Este proyecto de investigación intenta realizar la aplicación de nuevas líneas de investigación al programa de Comercio Internacional de la Universidad Francisco de Paula Santander UFPS. Fue financiado por la misma universidad, dentro del Grupo de Investigación para el Desarrollo Socioeconómico - GIDSE

El artículo está dividido en cuatro secciones. La primera sección presenta la metodología de la investigación. La segunda, el marco teórico de la construcción de las líneas de investigación. La tercera, los resultados a través de la identificación de las líneas de investigación para el Programa de Comercio Internacional de la Universidad Francisco de Paula Santander UFPS. Y la cuarta, presenta las conclusiones de la investigación.

El propósito principal de este artículo es construir líneas de investigación aplicables al Programa de Comercio Internacional de la Universidad
Francisco de Paula Santander, como apoyo al fortalecimiento del Programa y a las políticas de investigación de la Universidad. Para lograrlo se tuvieron en cuenta los siguientes objetivos específicos:

- Revisar las líneas de investigación de los diferentes grupos de investigación de la Facultad de Ciencias Empresariales de la UFPS.

- Definir la pertinencia en los temas de investigación de comercio internacional.

- Determinar las nuevas líneas de investigación al Programa de Comercio Internacional.

\section{Metodología}

En este tema de construcción de líneas de investigación se consideró el método inductivo que conlleva un análisis ordenado, coherente y lógico del problema de investigación, tomando como premisas verdaderas verdades particulares para concluir verdades generales (Méndez, 2006).

El nivel de investigación será de tipo descriptivo, cuyo propósito es la delimitación de los hechos que conforman el problema de investigación. El diseño de la investigación es de tipo documental y de campo. Documental porque se realizó a través de la consulta de documentos como el Proyecto Educativo del Programa - PEP, las resoluciones y acuerdos en materia de investigación de la Universidad y la documentación de las diferentes líneas de investigación de las universidades con programas a fines a comercio internacional.

El estudio de campo se realizó mediante encuestas que se aplicaron a empresarios de la ciudad de San José de Cúcuta y a los docentes del Programa de Comercio Internacional para determinar aspectos importantes del estudio sobre las líneas de investigación.

La población objeto de la investigación fue las empresas dedicadas a la importación, exportación y a las actividades de agenciamiento 
aduanero, logística, depósitos, agentes de carga y transporte de la ciudad de Cúcuta, además a los docentes del área de comercio internacional de la Universidad Francisco de Paula Santander. Por tanto, se tuvieron en cuenta las muestras de 62 empresarios del sector externo de la ciudad de San José de Cúcuta y 10 encuestas realizadas a los docentes del Programa de Comercio Internacional de la Universidad Francisco de Paula Santander.

\section{Marco teórico}

\section{a. Antecedentes}

Para la investigación se revisó literatura a nivel internacional, nacional y regional; considerando la esencia de cada una ellas, se destacan a continuación: el trabajo describe en síntesis la complejidad en que se tejen las relaciones al tema de las drogas; luego ubica la farmacodependencia como campo del conocimiento interdisciplinar, delimitando espacios con las ciencias y las disciplinas, y por último esta reflexión tiene por necesidad la construcción de una línea de investigación que centralice la producción en este campo del conocimiento, Castaño y Calderón (2004).

Sarmiento (2009), describe que pretende ser un documento estratégico y argumentativo, que actúe como directiva conceptual y metodológica para orientar la investigación contable y que aporte elementos para la formulación, asesoría, evaluación y validación de proyectos de investigación, así como criterios para la diseminación y publicación de sus resultados en el contexto latinoamericano.

Duque (2007), establece las dos sublíneas de investigación del Programa de Negocios Internacionales, las cuales son: 1). Productividad y competitividad y 2). Comercio internacional. Dentro de la compilación de este documento inicialmente establecen los criterios de las líneas de investigación de la Escuela de Administración y Mercadotecnia del Quindío - EAM; presenta los grupos de investigación de dicho programa y las líneas a seguir por ellos.

Morales (2001) realiza una revisión de la investigación en los tres programas de ingeniería que dispone la Universidad para las fuerzas militares. De esta manera, hacen una definición y comparación entre las áreas y las líneas de investigación. Presentan cinco áreas de investigación: la institucional; la básica científica; Geotécnica y construcción; vías, tránsito y transporte; y sanitaria y ambiental.

\section{b. Bases teóricas}

Para el desarrollo de esta investigación se hizo una revisión de algunas teorías en investigación y en economía, como son: Diseño y desarrollo del proceso de investigación con énfasis en ciencias empresariales; Comercio Internacional y Construcción de líneas de investigación

En el diseño y desarrollo del proceso de investigación con énfasis en ciencias empresariales, (Méndez 2006) establece que durante muchos años los programas de formación en las ciencias económicas, administrativas, contables, empresariales, de la salud y otras ciencias, han incluido en su currículo académico al menos un curso de investigación; al culminar el programa curricular, los estudiantes proceden al diseño y la elaboración de un trabajo de investigación para optar a su grado profesional. Cuando el estudiante inicia este último proceso encuentra problemas para la formulación de dicho trabajo, en especial en relación con el diseño, aspecto determinante en el contenido y la calidad de los trabajos propuestos.

El concepto de comercio internacional consiste en las transacciones de bienes y servicios entre países; viene del latín Commercium. El comercio es una actividad caracterizada por la compra o venta de bienes para su transformación o reventa. Internacional, es lo referente a dos o más países. De esta manera, las dos definiciones conllevan a la 
noción de que comercio internacional es una actividad comercial entre dos o más países.

El término de comercio internacional para algunos autores es referenciado como comercio exterior o comercio mundial. El comercio internacional junto con las finanzas internacionales hace parte de los dos grandes campos de la economía internacional. "El análisis del comercio internacional pone el énfasis en las transacciones reales de la economía internacional, es decir, en aquellas transacciones que implican un movimiento físico de bienes o un compromiso tangible de recursos económicos" (Krugman, Obstfeld y Melitz, 2012, p. 8)

El comercio internacional tiene diferentes teorías que explican su comportamiento: Teoría mercantilista; teoría de la ventaja absoluta; teoría de la ventaja relativa; teoría de la ventaja comparativa y competitiva.

En lo relacionado a la construcción de líneas de investigación, en las últimas décadas se le ha dado relevancia al tema de la investigación y de las líneas de investigación en las instituciones no solo de nivel básico, secundario o medio sino también el del orden superior como las universidades. Este es un tema que se ha venido observando desde los grados muy pequeños como lo son jardín y transición.

De esta manera, se observa mucha literatura en torno al tema de las áreas, las líneas y los grupos de investigación, ya sean dentro de los programas o facultades de las universidades tanto a nivel nacional como internacional. Es así como lo establece Cerda (2004) la línea de investigación se ha convertido en un auténtico vector de la investigación, es decir en un medio que no solo tiene magnitud, sentido, fuerza, aplicación y dirección, al igual que un vector físico o químico, sino capacidad para ordenar y estructurar los diversos componentes que participan en una investigación.

Otro aporte sobre algunas tareas prioritarias para la construcción de líneas de investigación es la Guía didáctica y módulo programas y líneas de investigación de la Fundación Universitaria Luis Amigo, realizada por Arboleda, Ramírez y Morales (2005).

La definición y fundamentación de una estrategia organizativa de investigación por Líneas de trabajo necesariamente deberá considerar la realización de una revisión, un reconocimiento y una apropiación crítica y sistemática de lo que hay construido y se esté construyendo hasta el momento de su formulación. Esto incluye los órdenes tanto de lo teórico como de lo experiencial que, como resultado, permita una visualización clara de las temáticas, objetos de estudio y problemáticas de los que se ocupará la Línea dentro del Campo de conocimiento elegido. (p.103)

\section{Resultados y discusión}

Después de haber revisado los criterios del Proyecto Educativo del Programa - PEP del Programa de Comercio Internacional sobre el tema de investigación, de analizar la encuesta realizada a los empresarios del sector exportador y las conclusiones del proyecto de investigación para la creación del Observatorio de Comercio Internacional; Además de realizar el estudio a la encuesta aplicada a los docentes del Programa de Comercio Internacional y la exploración de las líneas de investigación de las diferentes universidades colombianas con programas similares a comercio internacional, se intentó construir las líneas de investigación del Programa, pero esta construcción toma como referencia principal la línea de investigación del Grupo de Investigación para el Desarrollo Socioeconómico - GIDSE, Ciencias económicas y estudios internacionales.

De esta manera, se plantean las siguientes diez líneas de investigación: 
1. Estrategias de internacionalización de las empresas. Comprenderá los siguientes núcleos temáticos:

- Análisis del comportamiento de los sectores productivos exportadores de la región, establecer sus tendencias generales e identificar sus estrategias de internacionalización.

- Elaboración de planes de negocio a empresas exportadoras o importadoras.

- Los tratados de libre comercio, acuerdos comerciales y las oportunidades de las zonas francas.

2. Análisis del comercio internacional de Cúcuta y de Norte de Santander. La cual comprenderá:

- Análisis de las exportaciones e importaciones de la ciudad y de la región.

- La relación comercial Cúcuta - Venezuela.

- Los problemas económicos y sociales de la región de Norte de Santander y Venezuela.

3. Análisis de los sectores productivos de Cúcuta y Norte de Santander con miras al comercio internacional. Temas de estudio como son:

- La competitividad y productividad de bienes y servicios.

- El desarrollo industrial y la política industrial hacia la ciudad de Cúcuta.

- El sector de los servicios en el comercio internacional.

4. Desarrollo económico regional y local de Norte de Santander y de Santander. El núcleo temático de esta línea es:

- Identificar, promover y desarrollar elementos que contribuyan al crecimiento de la ciudad y de la región, como pueden ser las alianzas estratégicas, clúster, cadenas productivas.

- Construir e identificar los factores claves del crecimiento local y regional.

- Identificar y construir indicadores económicos y de comercio exterior de la región.

- Aplicar el uso de los recursos regionales de una forma apropiada y con un criterio sostenible con miras al comercio exterior.

- Aportes de la cooperación internacional a la región.

5. Logística y negocios internacionales. Lo relacionado a temas de:

- Análisis de operaciones.

- Negociación y comercio internacional.

- Logística estratégica.

- Migraciones.

6. Teoría del comercio y Políticas Comerciales. Se tienen en cuenta los núcleos temáticos como son:

- Teorías del comercio internacional.

- El Programa de Doha para el Desarrollo y las negociaciones en la OMC.

- Papel de las políticas comerciales internacionales y nacionales en el comercio internacional de la frontera.

- Aplicabilidad de la política comercial internacional y nacional en la región.

- Subnacionalesyorganismos internacionales

7. Contratación internacional y comercio electrónico. Se trabajan las siguientes áreas de estudio:

- Resolución de controversias en comercio internacional y en la negociación y redacción de los contratos internacionales más usuales (compraventa de mercaderías, 
agencia comercial, distribución comercial, franquicia, investigación y desarrollo y joint venture).

- Licitaciones internacionales.

- La empresa en la red.

- Seguridad jurídica de los negocios en internet.

8. La inversión extranjera directa y portafolio. Tendrá en cuenta los siguientes elementos:

- Alianzas de riesgo compartido.

- Franquicias.

- Adquisición de empresas existentes y montajes de empresas.

9. Gestión ambiental y tecnológica en el comercio internacional

- Economía y comercio verde.

- Innovación y tecnología de las empresas de la región con miras a mercados internacionales.

10. Finanzas y Relaciones Internacionales. Se revisarán los siguientes temas:

- Mercados financieros.

- Economías emergentes.

- Mercado de divisas.

- Relaciones internacionales.

Estas diez líneas de investigación se pueden sintetizar y corroborar en la figura 1:

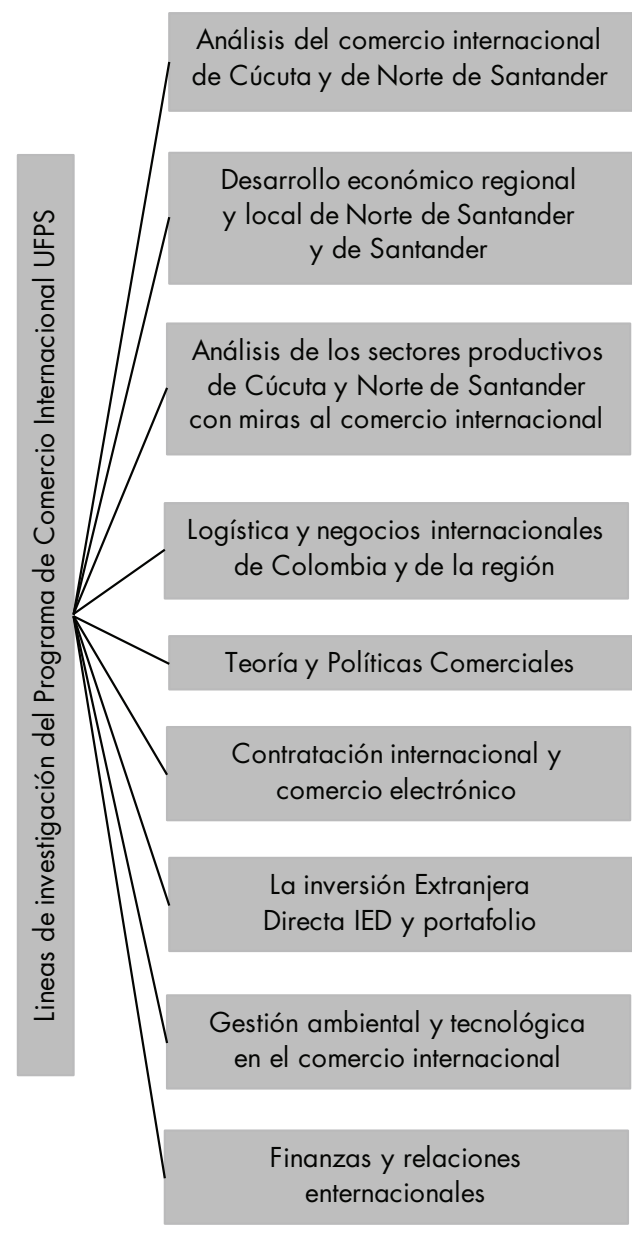

Figura 1. Líneas de investigación del Programa de Comercio Internacional de la UFPS

Fuente: Elaboración propia.

\section{Conclusiones}

Con la realización de este proyecto de investigación se pueden determinar las líneas de Investigación del Programa de Comercio Internacional de la Universidad Francisco de Paula Santander, teniendo en cuenta como eje central el Grupo de Investigación para el Desarrollo Socioeconómico GIDSE y una de las líneas de investigación del mismo, ciencias económicas y estudios internacionales.

Para la construcción de las líneas del Programa, se tomó como apoyo el Proyecto Educativo del Programa de Comercio Internacional - PEP 2014, la encuesta realizada para la definición de las áreas 
temáticas del Observatorio de Comercio Internacional - UFPS a los empresarios del sector externo de Cúcuta. También se tuvieron en cuenta los resultados de la encuesta aplicada a los docentes del Programa de Comercio Internacional - UFPS y la revisión documental de las líneas de investigación a seis universidades colombianas cuyos programas son afines al Programa de Comercio Internacional, como son las universidades: del Valle, Medellín, de la Sabana, Libre de Colombia, del Rosario y Sergio Arboleda.

A partir de este análisis, se realizó la construcción de diez líneas de investigación para el Programa de Comercio Internacional de la UFPS:

1. Estrategias de internacionalización de las empresas.

2. Análisis del comercio internacional de Cúcuta y de Norte de Santander.

3. Análisis de los sectores productivos de Cúcuta y Norte de Santander con miras al Comercio Internacional.

4. Desarrollo económico regional y local de Norte de Santander y de Santander.

5. Logística y negocios internacionales de Colombia y de la región.

6. Teoría y políticas comerciales.

7. Contratación internacional y comercio electrónico.

8. La inversión extranjera directa y portafolio.

9. Gestión ambiental y tecnológica en el comercio internacional

10. Finanzas y relaciones internacionales.

\section{Referencias}

Arboleda, O., Ramírez, P. y Morales, S (2005). Guía Didáctica y módulo programas y líneas de investigación. Fundación Universitaria Luis Amigo, Medellín, Colombia. Disponible en: http://virtual. funlam.edu.co/repositorio/sites/default/files/re positorioarchivos/2011/02/0054programasyline as.775.pdf

Calderón, R. \& Bayona, M. (2014). Estructuración del Observatorio de Comercio Internacional en la unidad de emprendimiento INNOVA UFPS. Universidad Francisco de Paula Santander, Cúcuta, Colombia.

Castaño, G. y Calderón, G. (2005). Reflexiones en relación a la construcción de líneas de investigación en Farmacodependencia. Universidad Federal de Santa Catarina, Florianópolis, Brasil.

Cerda, H. (2004). Hacia la Construcción de una línea de investigación. Seminario Taller. Universidad Cooperativa de Colombia. Bogotá: Editorial Teoría del Color Ltda.

Duque, A. (2007). Sublíneas de Investigación programa de negocios internacionales. Escuela de Administración y Mercadotecnia del Quindío, Armenia, Colombia. Disponible en: http://www.eam.edu. co/investigaciones/sites/default/files/sublineas_ negociosinternacionales.pdf

García C. y Núñez R. (2006). La formación en líneas de investigación, un tránsito de la información a la transformación. Manizales, Colombia.

Krugman P., Obstfeld M. y Melitz M. (2012). Economía Internacional: Teoría y política. Madrid España: Ediciones Pearson 9 edición.

Méndez, C. (2006) Metodología, diseño y desarrollo del proceso de investigación. México, Editorial Limusa.

Morales, L. (2001) Definición de las áreas y líneas de investigación de la Facultad de Ingeniería. Universidad Militar Nueva Granada, Bogotá, Colombia.

Sarmiento, H. (2009). Metodología para la formulación conceptual de líneas de investigación contable. Universidad Politécnico Colombiano, Bogotá. 